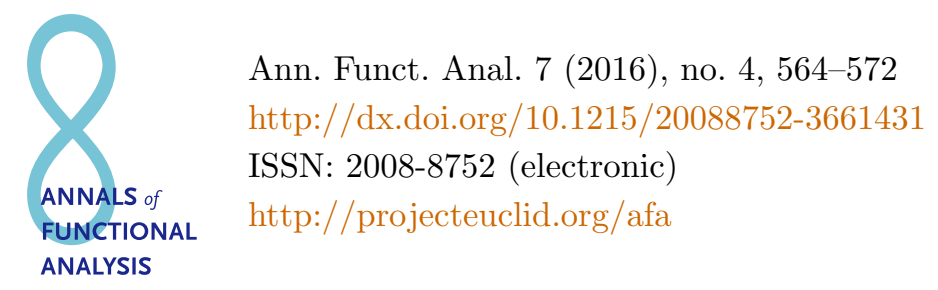

\title{
CHARACTER AMENABILITY AND CONTRACTIBILITY OF SOME BANACH ALGEBRAS ON LEFT COSET SPACES
}

\author{
M. RAMEZANPOUR, ${ }^{*}$ N. TAVALLAEI, and B. OLFATIAN GILLAN \\ Communicated by V. Runde
}

\begin{abstract}
Let $H$ be a compact subgroup of a locally compact group $G$, and let $\mu$ be a strongly quasi-invariant Radon measure on the homogeneous space $G / H$. In this article, we show that every element of $\widehat{G / H}$, the character space of $G / H$, determines a nonzero multiplicative linear functional on $L^{1}(G / H, \mu)$. Using this, we prove that for all $\phi \in \widehat{G / H}$, the right $\phi$-amenability of $L^{1}(G / H, \mu)$ and the right $\phi$-amenability of $M(G / H)$ are both equivalent to the amenability of $G$. Also, we show that $L^{1}(G / H, \mu)$, as well as $M(G / H)$, is right $\phi$-contractible if and only if $G$ is compact. In particular, when $H$ is the trivial subgroup, we obtain the known results on group algebras and measure algebras.
\end{abstract}

\section{INTRODUCTION}

Let $A$ be a Banach algebra and let $\Delta(A)$ be the spectrum of $A$, consisting of all nonzero multiplicative linear functionals on $A$. Then for $\varphi \in \Delta(A)$, the Banach algebra $A$ is called right $\varphi$-amenable if there exists an element $m \in A^{* *}$ satisfying $m(\varphi)=1$ and $m(f \cdot a)=\varphi(a) m(f)$ for all $a \in A$ and $f \in A^{*}$. One may similarly define the left $\varphi$-amenable Banach algebras. The right $\varphi$-amenability, as a modification of Johnson's amenability, was introduced and studied by Kaniuth, Lau, and Pym [7] under the name of $\varphi$-amenability. This notion of amenability is a generalization of the left amenability of a class of Banach algebras studied by Lau in [8] known as Lau algebras.

Copyright 2016 by the Tusi Mathematical Research Group.

Received Dec. 16, 2015; Accepted Mar. 20, 2016.

${ }^{*}$ Corresponding author.

2010 Mathematics Subject Classification. Primary 43A20; Secondary 46H05, 43A07.

Keywords. Banach algebra, homogeneous space, character amenability. 


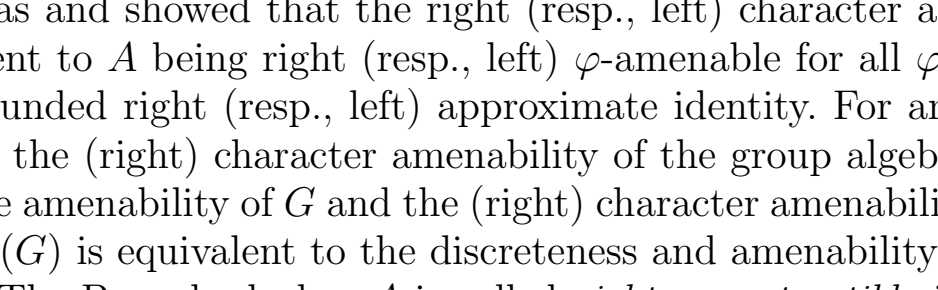

\title{
CHARACTER AMENABILITY AND CONTRACTIBILITY OF SOME BANACH ALGEBRAS ON LEFT COSET SPACES
}

\author{
M. RAMEZANPOUR, ${ }^{*}$ N. TAVALLAEI, and B. OLFATIAN GILLAN \\ Communicated by V. Runde
}

\begin{abstract}
Let $H$ be a compact subgroup of a locally compact group $G$, and let $\mu$ be a strongly quasi-invariant Radon measure on the homogeneous space $G / H$. In this article, we show that every element of $\widehat{G / H}$, the character space of $G / H$, determines a nonzero multiplicative linear functional on $L^{1}(G / H, \mu)$. Using this, we prove that for all $\phi \in \widehat{G / H}$, the right $\phi$-amenability of $L^{1}(G / H, \mu)$ and the right $\phi$-amenability of $M(G / H)$ are both equivalent to the amenability of $G$. Also, we show that $L^{1}(G / H, \mu)$, as well as $M(G / H)$, is right $\phi$-contractible if and only if $G$ is compact. In particular, when $H$ is the trivial subgroup, we obtain the known results on group algebras and measure algebras.
\end{abstract}

\section{INTRODUCTION}

Let $A$ be a Banach algebra and let $\Delta(A)$ be the spectrum of $A$, consisting of all nonzero multiplicative linear functionals on $A$. Then for $\varphi \in \Delta(A)$, the Banach algebra $A$ is called right $\varphi$-amenable if there exists an element $m \in A^{* *}$ satisfying $m(\varphi)=1$ and $m(f \cdot a)=\varphi(a) m(f)$ for all $a \in A$ and $f \in A^{*}$. One may similarly define the left $\varphi$-amenable Banach algebras. The right $\varphi$-amenability, as a modification of Johnson's amenability, was introduced and studied by Kaniuth, Lau, and Pym [7] under the name of $\varphi$-amenability. This notion of amenability is a generalization of the left amenability of a class of Banach algebras studied by Lau in [8] known as Lau algebras.

Copyright 2016 by the Tusi Mathematical Research Group.

Received Dec. 16, 2015; Accepted Mar. 20, 2016.

${ }^{*}$ Corresponding author.

2010 Mathematics Subject Classification. Primary 43A20; Secondary 46H05, 43A07.

Keywords. Banach algebra, homogeneous space, character amenability. 


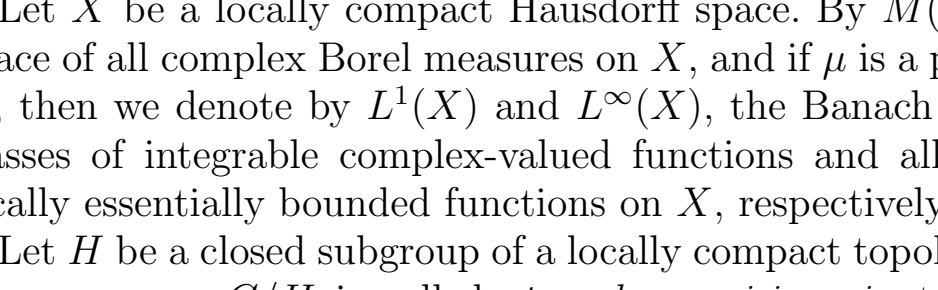

\title{
CHARACTER AMENABILITY AND CONTRACTIBILITY OF SOME BANACH ALGEBRAS ON LEFT COSET SPACES
}

\author{
M. RAMEZANPOUR, ${ }^{*}$ N. TAVALLAEI, and B. OLFATIAN GILLAN \\ Communicated by V. Runde
}

\begin{abstract}
Let $H$ be a compact subgroup of a locally compact group $G$, and let $\mu$ be a strongly quasi-invariant Radon measure on the homogeneous space $G / H$. In this article, we show that every element of $\widehat{G / H}$, the character space of $G / H$, determines a nonzero multiplicative linear functional on $L^{1}(G / H, \mu)$. Using this, we prove that for all $\phi \in \widehat{G / H}$, the right $\phi$-amenability of $L^{1}(G / H, \mu)$ and the right $\phi$-amenability of $M(G / H)$ are both equivalent to the amenability of $G$. Also, we show that $L^{1}(G / H, \mu)$, as well as $M(G / H)$, is right $\phi$-contractible if and only if $G$ is compact. In particular, when $H$ is the trivial subgroup, we obtain the known results on group algebras and measure algebras.
\end{abstract}

\section{INTRODUCTION}

Let $A$ be a Banach algebra and let $\Delta(A)$ be the spectrum of $A$, consisting of all nonzero multiplicative linear functionals on $A$. Then for $\varphi \in \Delta(A)$, the Banach algebra $A$ is called right $\varphi$-amenable if there exists an element $m \in A^{* *}$ satisfying $m(\varphi)=1$ and $m(f \cdot a)=\varphi(a) m(f)$ for all $a \in A$ and $f \in A^{*}$. One may similarly define the left $\varphi$-amenable Banach algebras. The right $\varphi$-amenability, as a modification of Johnson's amenability, was introduced and studied by Kaniuth, Lau, and Pym [7] under the name of $\varphi$-amenability. This notion of amenability is a generalization of the left amenability of a class of Banach algebras studied by Lau in [8] known as Lau algebras.

Copyright 2016 by the Tusi Mathematical Research Group.

Received Dec. 16, 2015; Accepted Mar. 20, 2016.

${ }^{*}$ Corresponding author.

2010 Mathematics Subject Classification. Primary 43A20; Secondary 46H05, 43A07.

Keywords. Banach algebra, homogeneous space, character amenability. 


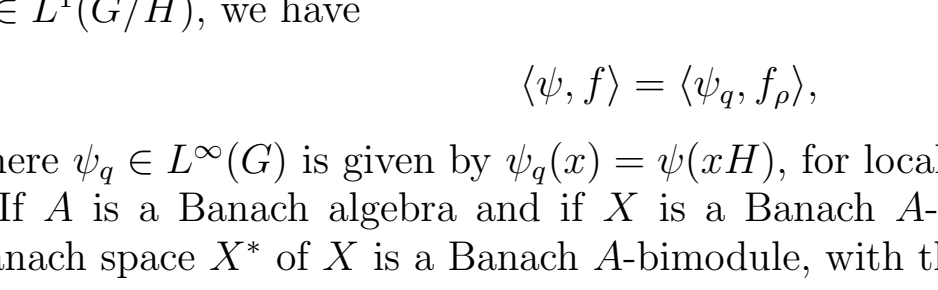

\title{
CHARACTER AMENABILITY AND CONTRACTIBILITY OF SOME BANACH ALGEBRAS ON LEFT COSET SPACES
}

\author{
M. RAMEZANPOUR, ${ }^{*}$ N. TAVALLAEI, and B. OLFATIAN GILLAN \\ Communicated by V. Runde
}

\begin{abstract}
Let $H$ be a compact subgroup of a locally compact group $G$, and let $\mu$ be a strongly quasi-invariant Radon measure on the homogeneous space $G / H$. In this article, we show that every element of $\widehat{G / H}$, the character space of $G / H$, determines a nonzero multiplicative linear functional on $L^{1}(G / H, \mu)$. Using this, we prove that for all $\phi \in \widehat{G / H}$, the right $\phi$-amenability of $L^{1}(G / H, \mu)$ and the right $\phi$-amenability of $M(G / H)$ are both equivalent to the amenability of $G$. Also, we show that $L^{1}(G / H, \mu)$, as well as $M(G / H)$, is right $\phi$-contractible if and only if $G$ is compact. In particular, when $H$ is the trivial subgroup, we obtain the known results on group algebras and measure algebras.
\end{abstract}

\section{INTRODUCTION}

Let $A$ be a Banach algebra and let $\Delta(A)$ be the spectrum of $A$, consisting of all nonzero multiplicative linear functionals on $A$. Then for $\varphi \in \Delta(A)$, the Banach algebra $A$ is called right $\varphi$-amenable if there exists an element $m \in A^{* *}$ satisfying $m(\varphi)=1$ and $m(f \cdot a)=\varphi(a) m(f)$ for all $a \in A$ and $f \in A^{*}$. One may similarly define the left $\varphi$-amenable Banach algebras. The right $\varphi$-amenability, as a modification of Johnson's amenability, was introduced and studied by Kaniuth, Lau, and Pym [7] under the name of $\varphi$-amenability. This notion of amenability is a generalization of the left amenability of a class of Banach algebras studied by Lau in [8] known as Lau algebras.

Copyright 2016 by the Tusi Mathematical Research Group.

Received Dec. 16, 2015; Accepted Mar. 20, 2016.

${ }^{*}$ Corresponding author.

2010 Mathematics Subject Classification. Primary 43A20; Secondary 46H05, 43A07.

Keywords. Banach algebra, homogeneous space, character amenability. 


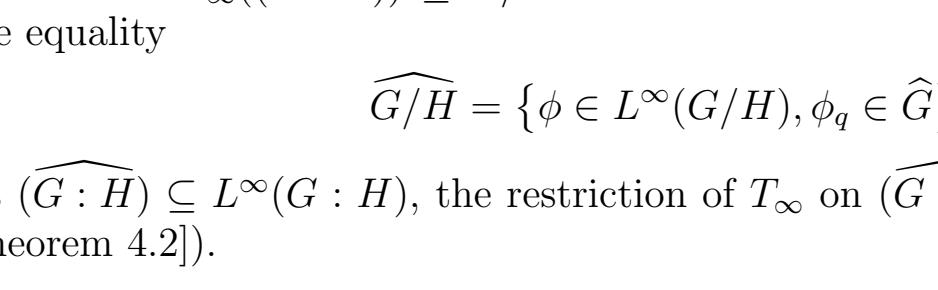

\title{
CHARACTER AMENABILITY AND CONTRACTIBILITY OF SOME BANACH ALGEBRAS ON LEFT COSET SPACES
}

\author{
M. RAMEZANPOUR, ${ }^{*}$ N. TAVALLAEI, and B. OLFATIAN GILLAN \\ Communicated by V. Runde
}

\begin{abstract}
Let $H$ be a compact subgroup of a locally compact group $G$, and let $\mu$ be a strongly quasi-invariant Radon measure on the homogeneous space $G / H$. In this article, we show that every element of $\widehat{G / H}$, the character space of $G / H$, determines a nonzero multiplicative linear functional on $L^{1}(G / H, \mu)$. Using this, we prove that for all $\phi \in \widehat{G / H}$, the right $\phi$-amenability of $L^{1}(G / H, \mu)$ and the right $\phi$-amenability of $M(G / H)$ are both equivalent to the amenability of $G$. Also, we show that $L^{1}(G / H, \mu)$, as well as $M(G / H)$, is right $\phi$-contractible if and only if $G$ is compact. In particular, when $H$ is the trivial subgroup, we obtain the known results on group algebras and measure algebras.
\end{abstract}

\section{INTRODUCTION}

Let $A$ be a Banach algebra and let $\Delta(A)$ be the spectrum of $A$, consisting of all nonzero multiplicative linear functionals on $A$. Then for $\varphi \in \Delta(A)$, the Banach algebra $A$ is called right $\varphi$-amenable if there exists an element $m \in A^{* *}$ satisfying $m(\varphi)=1$ and $m(f \cdot a)=\varphi(a) m(f)$ for all $a \in A$ and $f \in A^{*}$. One may similarly define the left $\varphi$-amenable Banach algebras. The right $\varphi$-amenability, as a modification of Johnson's amenability, was introduced and studied by Kaniuth, Lau, and Pym [7] under the name of $\varphi$-amenability. This notion of amenability is a generalization of the left amenability of a class of Banach algebras studied by Lau in [8] known as Lau algebras.

Copyright 2016 by the Tusi Mathematical Research Group.

Received Dec. 16, 2015; Accepted Mar. 20, 2016.

${ }^{*}$ Corresponding author.

2010 Mathematics Subject Classification. Primary 43A20; Secondary 46H05, 43A07.

Keywords. Banach algebra, homogeneous space, character amenability. 


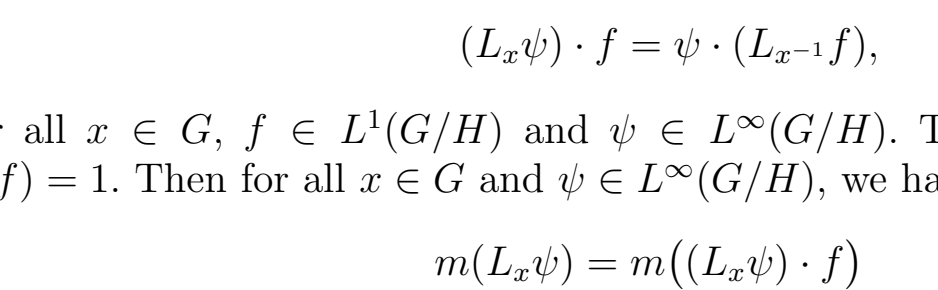

\title{
CHARACTER AMENABILITY AND CONTRACTIBILITY OF SOME BANACH ALGEBRAS ON LEFT COSET SPACES
}

\author{
M. RAMEZANPOUR, ${ }^{*}$ N. TAVALLAEI, and B. OLFATIAN GILLAN \\ Communicated by V. Runde
}

\begin{abstract}
Let $H$ be a compact subgroup of a locally compact group $G$, and let $\mu$ be a strongly quasi-invariant Radon measure on the homogeneous space $G / H$. In this article, we show that every element of $\widehat{G / H}$, the character space of $G / H$, determines a nonzero multiplicative linear functional on $L^{1}(G / H, \mu)$. Using this, we prove that for all $\phi \in \widehat{G / H}$, the right $\phi$-amenability of $L^{1}(G / H, \mu)$ and the right $\phi$-amenability of $M(G / H)$ are both equivalent to the amenability of $G$. Also, we show that $L^{1}(G / H, \mu)$, as well as $M(G / H)$, is right $\phi$-contractible if and only if $G$ is compact. In particular, when $H$ is the trivial subgroup, we obtain the known results on group algebras and measure algebras.
\end{abstract}

\section{INTRODUCTION}

Let $A$ be a Banach algebra and let $\Delta(A)$ be the spectrum of $A$, consisting of all nonzero multiplicative linear functionals on $A$. Then for $\varphi \in \Delta(A)$, the Banach algebra $A$ is called right $\varphi$-amenable if there exists an element $m \in A^{* *}$ satisfying $m(\varphi)=1$ and $m(f \cdot a)=\varphi(a) m(f)$ for all $a \in A$ and $f \in A^{*}$. One may similarly define the left $\varphi$-amenable Banach algebras. The right $\varphi$-amenability, as a modification of Johnson's amenability, was introduced and studied by Kaniuth, Lau, and Pym [7] under the name of $\varphi$-amenability. This notion of amenability is a generalization of the left amenability of a class of Banach algebras studied by Lau in [8] known as Lau algebras.

Copyright 2016 by the Tusi Mathematical Research Group.

Received Dec. 16, 2015; Accepted Mar. 20, 2016.

${ }^{*}$ Corresponding author.

2010 Mathematics Subject Classification. Primary 43A20; Secondary 46H05, 43A07.

Keywords. Banach algebra, homogeneous space, character amenability. 


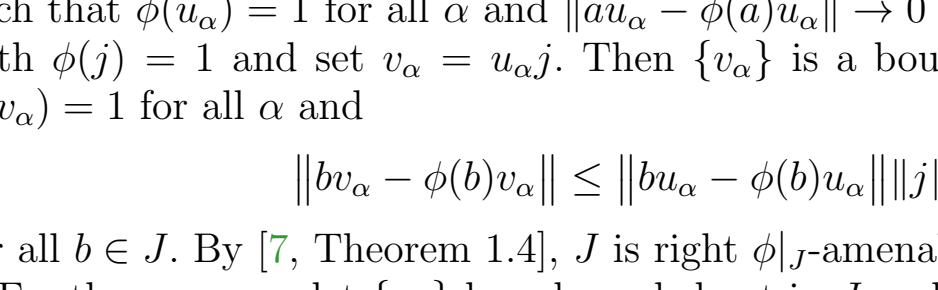

\title{
CHARACTER AMENABILITY AND CONTRACTIBILITY OF SOME BANACH ALGEBRAS ON LEFT COSET SPACES
}

\author{
M. RAMEZANPOUR, ${ }^{*}$ N. TAVALLAEI, and B. OLFATIAN GILLAN \\ Communicated by V. Runde
}

\begin{abstract}
Let $H$ be a compact subgroup of a locally compact group $G$, and let $\mu$ be a strongly quasi-invariant Radon measure on the homogeneous space $G / H$. In this article, we show that every element of $\widehat{G / H}$, the character space of $G / H$, determines a nonzero multiplicative linear functional on $L^{1}(G / H, \mu)$. Using this, we prove that for all $\phi \in \widehat{G / H}$, the right $\phi$-amenability of $L^{1}(G / H, \mu)$ and the right $\phi$-amenability of $M(G / H)$ are both equivalent to the amenability of $G$. Also, we show that $L^{1}(G / H, \mu)$, as well as $M(G / H)$, is right $\phi$-contractible if and only if $G$ is compact. In particular, when $H$ is the trivial subgroup, we obtain the known results on group algebras and measure algebras.
\end{abstract}

\section{INTRODUCTION}

Let $A$ be a Banach algebra and let $\Delta(A)$ be the spectrum of $A$, consisting of all nonzero multiplicative linear functionals on $A$. Then for $\varphi \in \Delta(A)$, the Banach algebra $A$ is called right $\varphi$-amenable if there exists an element $m \in A^{* *}$ satisfying $m(\varphi)=1$ and $m(f \cdot a)=\varphi(a) m(f)$ for all $a \in A$ and $f \in A^{*}$. One may similarly define the left $\varphi$-amenable Banach algebras. The right $\varphi$-amenability, as a modification of Johnson's amenability, was introduced and studied by Kaniuth, Lau, and Pym [7] under the name of $\varphi$-amenability. This notion of amenability is a generalization of the left amenability of a class of Banach algebras studied by Lau in [8] known as Lau algebras.

Copyright 2016 by the Tusi Mathematical Research Group.

Received Dec. 16, 2015; Accepted Mar. 20, 2016.

${ }^{*}$ Corresponding author.

2010 Mathematics Subject Classification. Primary 43A20; Secondary 46H05, 43A07.

Keywords. Banach algebra, homogeneous space, character amenability. 


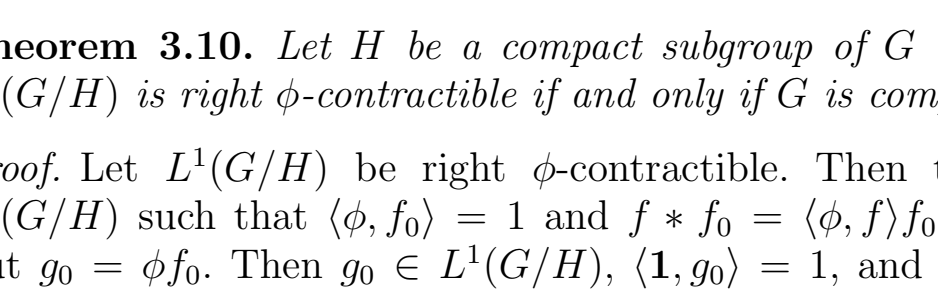

\title{
CHARACTER AMENABILITY AND CONTRACTIBILITY OF SOME BANACH ALGEBRAS ON LEFT COSET SPACES
}

\author{
M. RAMEZANPOUR, ${ }^{*}$ N. TAVALLAEI, and B. OLFATIAN GILLAN \\ Communicated by V. Runde
}

\begin{abstract}
Let $H$ be a compact subgroup of a locally compact group $G$, and let $\mu$ be a strongly quasi-invariant Radon measure on the homogeneous space $G / H$. In this article, we show that every element of $\widehat{G / H}$, the character space of $G / H$, determines a nonzero multiplicative linear functional on $L^{1}(G / H, \mu)$. Using this, we prove that for all $\phi \in \widehat{G / H}$, the right $\phi$-amenability of $L^{1}(G / H, \mu)$ and the right $\phi$-amenability of $M(G / H)$ are both equivalent to the amenability of $G$. Also, we show that $L^{1}(G / H, \mu)$, as well as $M(G / H)$, is right $\phi$-contractible if and only if $G$ is compact. In particular, when $H$ is the trivial subgroup, we obtain the known results on group algebras and measure algebras.
\end{abstract}

\section{INTRODUCTION}

Let $A$ be a Banach algebra and let $\Delta(A)$ be the spectrum of $A$, consisting of all nonzero multiplicative linear functionals on $A$. Then for $\varphi \in \Delta(A)$, the Banach algebra $A$ is called right $\varphi$-amenable if there exists an element $m \in A^{* *}$ satisfying $m(\varphi)=1$ and $m(f \cdot a)=\varphi(a) m(f)$ for all $a \in A$ and $f \in A^{*}$. One may similarly define the left $\varphi$-amenable Banach algebras. The right $\varphi$-amenability, as a modification of Johnson's amenability, was introduced and studied by Kaniuth, Lau, and Pym [7] under the name of $\varphi$-amenability. This notion of amenability is a generalization of the left amenability of a class of Banach algebras studied by Lau in [8] known as Lau algebras.

Copyright 2016 by the Tusi Mathematical Research Group.

Received Dec. 16, 2015; Accepted Mar. 20, 2016.

${ }^{*}$ Corresponding author.

2010 Mathematics Subject Classification. Primary 43A20; Secondary 46H05, 43A07.

Keywords. Banach algebra, homogeneous space, character amenability. 


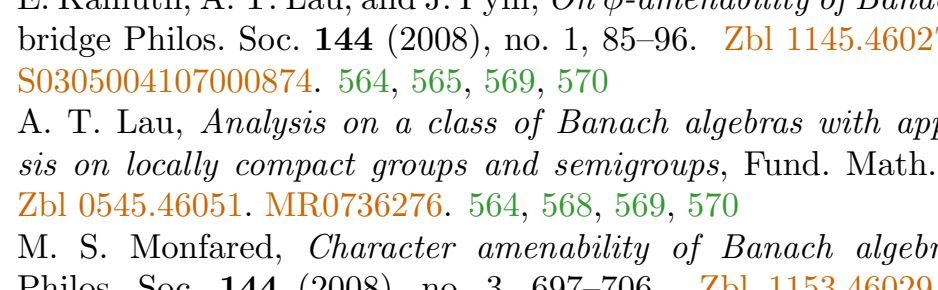

\title{
CHARACTER AMENABILITY AND CONTRACTIBILITY OF SOME BANACH ALGEBRAS ON LEFT COSET SPACES
}

\author{
M. RAMEZANPOUR, ${ }^{*}$ N. TAVALLAEI, and B. OLFATIAN GILLAN \\ Communicated by V. Runde
}

\begin{abstract}
Let $H$ be a compact subgroup of a locally compact group $G$, and let $\mu$ be a strongly quasi-invariant Radon measure on the homogeneous space $G / H$. In this article, we show that every element of $\widehat{G / H}$, the character space of $G / H$, determines a nonzero multiplicative linear functional on $L^{1}(G / H, \mu)$. Using this, we prove that for all $\phi \in \widehat{G / H}$, the right $\phi$-amenability of $L^{1}(G / H, \mu)$ and the right $\phi$-amenability of $M(G / H)$ are both equivalent to the amenability of $G$. Also, we show that $L^{1}(G / H, \mu)$, as well as $M(G / H)$, is right $\phi$-contractible if and only if $G$ is compact. In particular, when $H$ is the trivial subgroup, we obtain the known results on group algebras and measure algebras.
\end{abstract}

\section{INTRODUCTION}

Let $A$ be a Banach algebra and let $\Delta(A)$ be the spectrum of $A$, consisting of all nonzero multiplicative linear functionals on $A$. Then for $\varphi \in \Delta(A)$, the Banach algebra $A$ is called right $\varphi$-amenable if there exists an element $m \in A^{* *}$ satisfying $m(\varphi)=1$ and $m(f \cdot a)=\varphi(a) m(f)$ for all $a \in A$ and $f \in A^{*}$. One may similarly define the left $\varphi$-amenable Banach algebras. The right $\varphi$-amenability, as a modification of Johnson's amenability, was introduced and studied by Kaniuth, Lau, and Pym [7] under the name of $\varphi$-amenability. This notion of amenability is a generalization of the left amenability of a class of Banach algebras studied by Lau in [8] known as Lau algebras.

Copyright 2016 by the Tusi Mathematical Research Group.

Received Dec. 16, 2015; Accepted Mar. 20, 2016.

${ }^{*}$ Corresponding author.

2010 Mathematics Subject Classification. Primary 43A20; Secondary 46H05, 43A07.

Keywords. Banach algebra, homogeneous space, character amenability. 